\title{
Improving the Quality of Service Routing in OLSR Protocol
}

\author{
Dalil Moad ${ }^{\mp}$,Soufiene Djahel ${ }^{\ddagger}$ and Farid Naït-Abdesselam ${ }^{ \pm}$ \\ F CityPassenger SA, 1 Avenue de l'Atlantique,Les Conquérents BP 903, 91976 Courtaboeuf Cedex, France \\ ${ }^{ \pm}$University of Paris Descartes, 45, Rue des Saints-Peres, 75006 Paris, France \\ ${ }^{\ddagger}$ Lero, UCD School of Computer Science and Informatics, Ireland
}

\begin{abstract}
In this paper we propose an approach to improve the quality of service $(\mathrm{QoS})$ routing in the optimized link state routing protocol. The OLSR protocol operates generally in a best effort mode by finding the shortest path between a source and a destination without any quality of service consideration. We argue that an optimal path is not always the shortest path, and based on the network configuration and load, other alternatives such as a longer path with a high bandwidth might be of a better interest. To provide such alternatives and improve the communication quality among end users, our approach proposes to perform at each node an estimation of the bandwidth share between all adjacent nodes and tends to ensure the selection of a path with all MPRs that provide a higher bandwidth along the path. The bandwidth share estimation on each link is based on the study of conflict graphs to derive the set of maximal cliques. Once the bandwidth share estimation is done, instead of choosing the shortest path following the usual heuristic in OLSR, we try to find the path that ensures the highest bandwidth among all possible paths between the source node and the destination node. Results from simulation experiments show that the proposed approach achieves a higher performance than the standard OLSR used in wireless mesh networks.
\end{abstract}

Keywords - Wireless Mesh Networks, IEEE 802.11, Qality of Service, OLSR protocol, Conflict graph.

\section{INTRODUCTION}

Wireless Mesh Networks (WMNs) are a prominent technology of wireless communication that has seen a tremendous growth in the last few years. WMNs provide support for ad networking with capability of self-forming, self-healing, self-organizing as well as self-configuring [4]. WMNs have recently attracted a lot of attention from both academia and industry due to the above mentioned characteristics and their flexibility, their ease and reduced cost of deployment. A WMN is composed of both wired and wireless nodes forming a mesh topology. Special nodes named gateways are usually equipped with multiple interfaces (wired and wireless) and serve as Internet access points to the mesh clients. These gateways can be either stationary (e.g. rooftop) or mobile (e.g. airplane, buses/subway). In WMNs, a large number of Mesh routers is needed in order to provide a reliable service. Each router is equipped with at least one wireless interface and acts as a repeater to transmit data from nearby mesh routers/clients to farther mesh nodes. The mesh clients are the only sources/destinations of data traffic flows in the network.

Many routing protocols have been standardized by IETF for WMNs, among which the Optimized Link State Routing (OLSR) protocol [1]. OLSR is a proactive routing protocol proposed for Mobile Ad hoc NETworks (MANETs), it is an optimization of link state protocols. The key concept in OLSR is MPRs (Multipoint relays) which are responsible for spreading control traffic to the whole network via transmission of TC (Topology Control) messages. TC messages are exclusively sent by the MPR nodes, so this significantly reduce the incurred overhead during the flooding process. Moreover, the MPR nodes are used to construct the routing paths because any route in the network is computed using the information contained in TC messages. In OLSR, an optimal route relying two nodes is calculated based on the MPR selection mechanism defined in the RFC 3626 [1], in which the main routes selection criterion is the number of hops to the destination node.

The main drawback of OLSR is its lack of mechanisms that allow it to provide QoS guarantees, which makes it unable to find the optimal route that satisfies the QoS requirements of a given traffic flow. Moreover, we cannot apply this heuristic in QoS routing for MPR selection in OLSR due to their selection criteria which is the number of hops. This criteria may hide the good quality links to other nodes. To circumvent such drawback, we propose, in this paper, a new approach that ensures to find the path with the best QoS based on Bandwidth fair-share estimation between the adjacent nodes.

The remainder of the paper is organized as follows. Section II is devoted to the background and the related work on the MPR selection scheme in OLSR. The detailed description of our approach is, then, presented in Section III. The simulation results are presented and discussed in section IV. Finally, Section V concludes the paper.

\section{MPR SELECTION SCHEME IN OSLR STANDARD AND RELATED WORKS}

In wireless networks, finding the path that ensures a high throughput to fulfil the QoS requirements is essential given the instability of the medium of these networks compared to their wired counterparts. The most used metric in Ad hoc networks for routing is the minimum hops number. AODV(Ad hoc On Demand Distance Vector) [2] and OLSR [1] are an example of the routing protocols that use this metric. The use of the minimum hops number in these protocols is not a good 
idea since some links which are more stable and have higher bandwidth may not be chosen in the routing paths.

Recently, a number of schemes have been proposed to deal with routing issues in wireless mesh networks. In what follows, we summarize the most significant contributions in the literature.

In [3] De Couto et al. propose a new metric to select a path in multi-hop routing which is the expected transmission count (ETX) [3], this metric can improve the throughput of a wireless network but it is not designed to cope well with short-term channel variations because it uses the mean loss ratios to decide the routing. In [5], the authors have used Expected Transmission Time (ETT) as a metric for routing decisions, they improve ETX by considering the differences in the link transmission rate. The drawback of the above metrics is that they did not take into account the interference between adjacent nodes.

As opposed to the existing schemes in the literature, where the hop count is the main metric used to select the best routing path, we propose a novel MPR selection scheme that ensures a better QoS in WMNs using OLSR. In our scheme, we choose the set of MPR nodes based on the bandwidth fair share estimation among a set of neighbor nodes in order to guarantee that the chosen routing path ensures high bandwidth from the source node to the destination. The bandwidth estimation is carried out according to the proposed scheme in [7].

The heuristic for selection of MPRs set proposed in OLSR standard is given in flow chart in Figure. 2. The Optimized Link State Routing protocol (OLSR) [1] is a proactive routing protocol designed for Mobile Ad hoc networks (MANETs). It periodically sends control packets to build and update the topology of the network. MPRs (Multipoint relays) are the key concept in OLSR, which are a set of neighbor nodes that are responsible for spreading the local link state information to the whole network, they allow to reduce the induced overhead during the flooding process. The protocol is particularly suitable for large and dense networks as the technique of MPRs works well in this context. Notice that the local link state information is periodically spread to the whole network by the MPR nodes via the transmission of TC messages.

In OLSR, each node selects its MPR set among its one hop symmetric neighbors. These MPRs allow a node to reach all its two hops neighbors with minimum number of retransmissions. Spreading a message to the whole network with repetition can be done by classical flooding mechanism using the following rule: a node relays a message if and only if it has not already received it. Spreading a message using the MPR nodes reduces significantly the induced overhead, compared to the above spreading mechanism, due to the following rule: an MPR node relays a message if and only if : 1) it hasn't already received it, and 2) it just received it from a node which belongs to its MPR selectors set. Figure. 1 shows an example illustrating the MPR set selection mechanism. The node for which we want to compute the MPR set is B, the set of B's 1-hop neighbors is A,C,D,E,F. The set of B's 2-hop neighbors is G,H,I,J. Then, to reach this set we can pass through the nodes belonging to one of the following sub-sets: $\{\mathrm{D}, \mathrm{E}\},\{\mathrm{D}, \mathrm{F}\}$ or $\{\mathrm{D}, \mathrm{E}, \mathrm{F}\}$. Since we have two minimal sub-sets which are $\{\mathrm{D}, \mathrm{F}\}$ and $\{\mathrm{D}, \mathrm{F}\}$, then the chosen MPR set of B is $\{\mathrm{D}, \mathrm{E}\}$ because $\mathrm{E}$ has higher connectivity than $\mathrm{F}$ (i.e, $\mathrm{E}$ is connected to $\mathrm{H}, \mathrm{I}$, J however $\mathrm{F}$ is connected only to I and $\mathrm{J}$ ).

The selection of MPR is NP-complet and done according to the algorithm defined in RFC 3626 as described in the flowchart shown in Figure. 2. Notice that $N_{1}, N_{2}$ and $D(y)$ are described as follows.

- $N_{1}$ : 1-hop neighbors set of the node $\mathrm{X}$ which we want to determine its MPRs.

- $N_{2}$ : 2-hop neighbors set of node $X$. Using Hello message, all 1-hop neighbors of the node $X$ declare their 1hop neighbors. Thus, the node $X$ will know the 1-hop neighbors that must request to transmit a packet to its 2-hop neighbors. Moreover, the asymmetric links are detected, thanks to Hello messages, but not considered for MPR selection process.

- $D(y)$ : the degree of 1-hop neighbor node $\mathbf{y}$, is defined as the number of symmetric neighbors of node $y$, excluding all the members of $\mathrm{N}$ and $\mathrm{y}$.

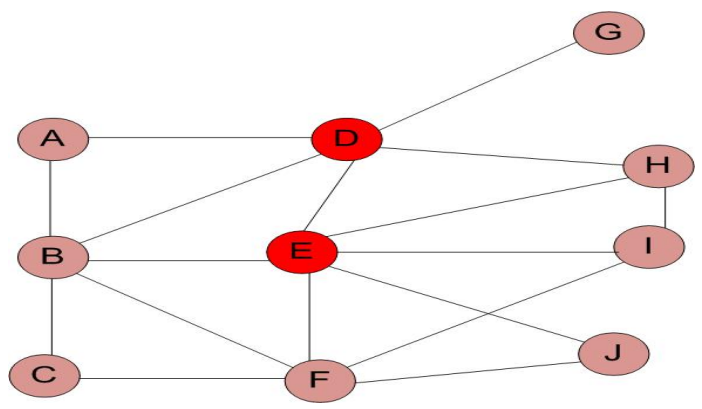

Figure 1: An example of network topology illustrating the MPR selection process: nodes $\mathrm{E}$ and $\mathrm{F}$ are MPRs for node $\mathrm{B}$

\section{THE PROPOSED SOLUTION}

In this section, we present our approach that aims to find the best route that guarantees the QoS requirements from the source to the destination node using bandwidth fair-share estimation between adjacent nodes along with the weights given to each link. Therefore, instead of choosing the shortest path, we try to find the path with the highest bandwidth. The key idea of our approach is to use the conflict graph to represent the interferences area and then calculate the maximal cliques in this graph. After extracting the set of maximal cliques, we estimate the bandwidth acquired by each link. To do so, two steps are needed as described below:

- Bandwidth fair-share estimation between adjacent nodes.

- Find the route which guarantees the best QoS in terms of bandwidth.

Finding the best route requires to design a new algorithm which allows to select the MPR set in OLSR using the bandwidth as the main criterion of selection. The flowchart 


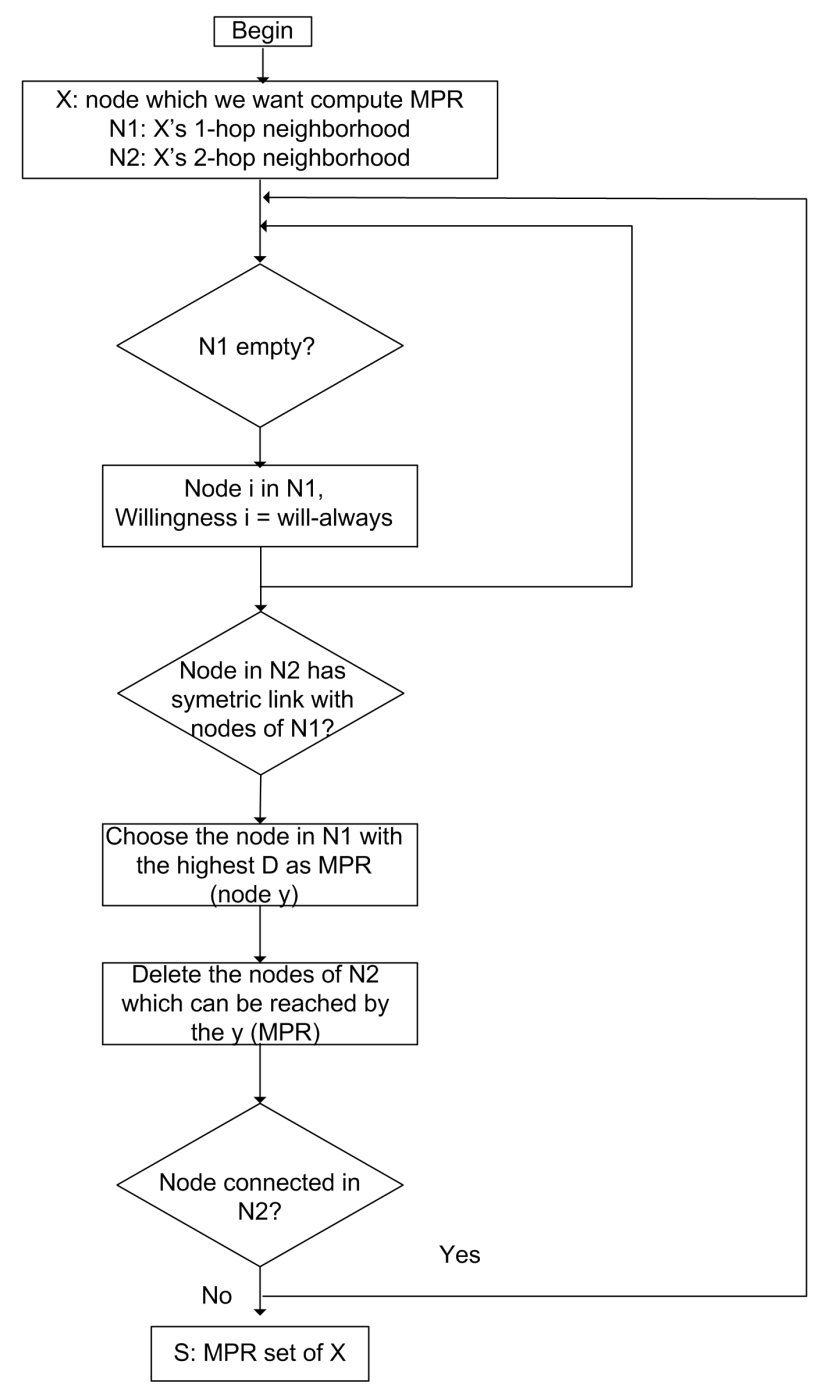

Figure 2: Flowchart of MPR nodes selection in OLSR standard

shown in Figure. 3 describes our proposed MPR selection scheme.

\section{A. Conflict graph construction}

As a first step of our approach, the node seeking for a path towards its destination constructs the contention flow graph with nodes within its carrier sensing range to derive its predicted fair-share of bandwidth according to the schemes proposed in [6] and [7].

After establishing the connectivity graph (transmission graph) between nodes, thanks to the analyze of the received information in Hello and TC messages, the graph is constructed by considering the worst case scenario ${ }^{1}$ assuming the maximum number of contending links to compute the bandwidth fair share. We represent the wireless interferences by a conflict graph [8]. For example, in Figure. 4 the node E acquires the set of its 2-hops neighbors $\mathrm{A}, \mathrm{B}, \mathrm{G}$ and $\mathrm{H}$ from

\footnotetext{
${ }^{1}$ The worst case scenario is considered because the topological information acquired from Hello and TC messages is partial. Thus, we haven't a clear vision about the rest of the links.
}

the Hello messages sent by the nodes $\mathrm{C}$ and F. Due to the TC message sent by $\mathrm{H}$ which is an MPR (Multipoint Relay) of I, the node $\mathrm{E}$ discovers that the node I is its 3-hops neighbors. After acquiring the necessary information for the last step, the node $\mathrm{E}$ builds the conflict graph within its carrier sensing range, whose vertices correspond to links in the connectivity graph, as shown in Figure. 4, from which it extracts the set of maximal cliques [9]. Since the topological information acquired is partial, node $\mathrm{E}$ builds this graph by assuming the maximum number of contending links.

The set of maximal cliques corresponding to the conflict graph in Figure. 5 is shown in Figure. 6:

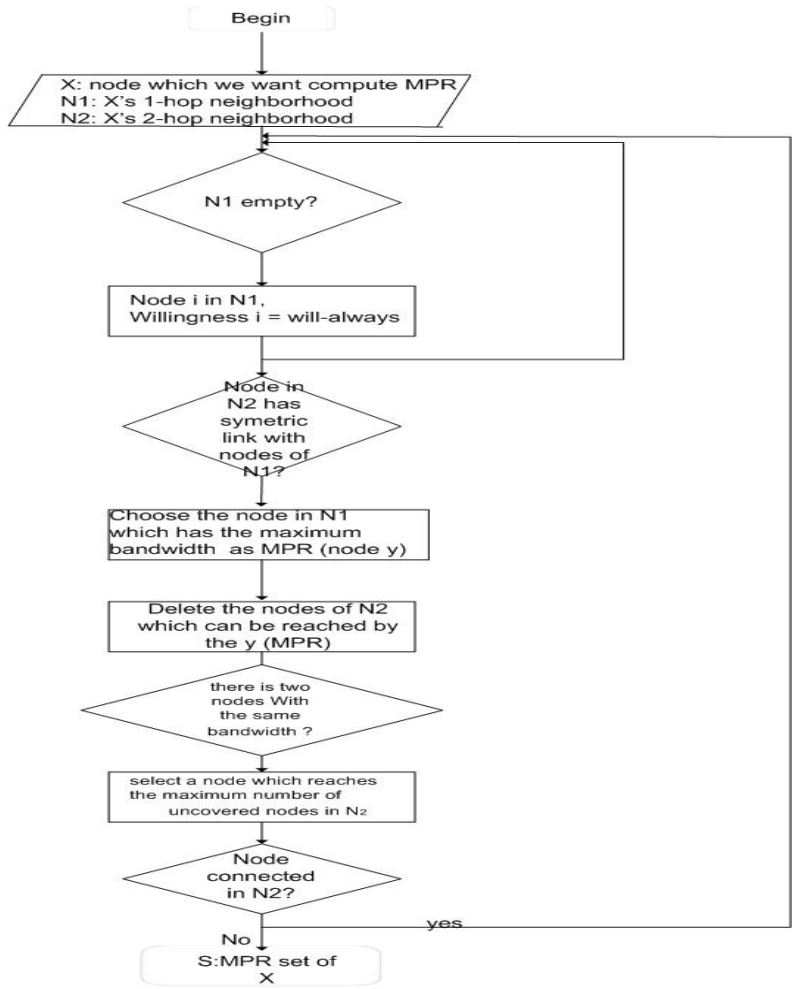

Figure 3: Flowchart of MPR selection with bandwidth constraints

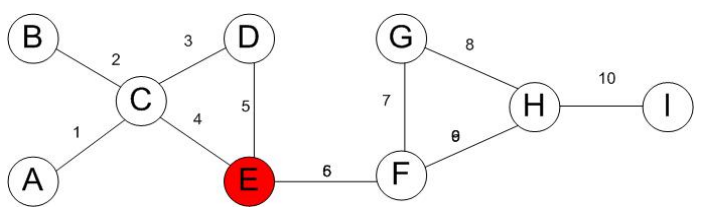

Figure 4: The connectivity graph

\section{B. How to estimate the bandwidth with the weights?}

Once the conflict graph is established and the set of maximal cliques is derived, the node $\mathrm{E}$ estimates its fair-share of bandwidth as follows.

For each clique, we compute the weight corresponding to the links that compose it. For example, in Figure. 6, we found three maximal cliques whose sizes are 5, 5 and 6 , 


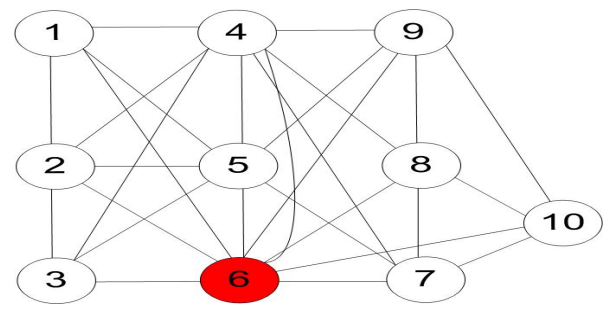

Figure 5: The conflict graph
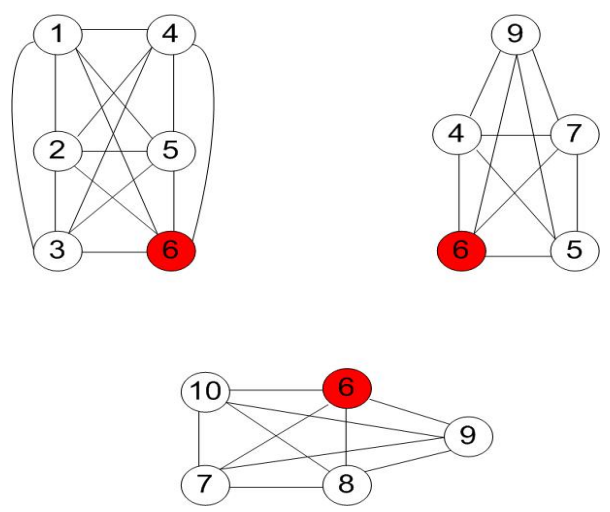

Figure 6: The set of maximal cliques

respectively. The weight for the nodes belonging to both of theses cliques with size 5 is $1 / 5$ and for the third clique is $1 / 6$. If a node belongs to more than one clique we choose the minimum weight (1). The connectivity graph with weights is represented in Figure. 7 whose the weights are in red color.

Let $W(j)$ the weight of the node $j$.

$n$ : number of maximal cliques that contain the node $j$.

$W(j)=\min _{i=1}^{n} W_{i}(j)$

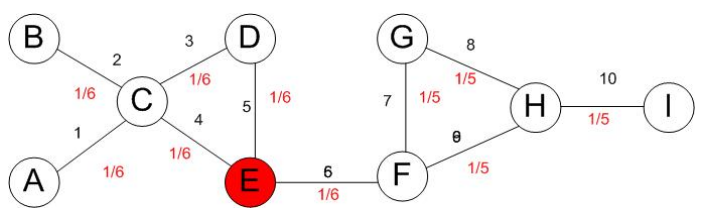

Figure 7: Connectivity graph with weights

\section{Route selection under QoS constraints}

1) First step: The first step consists in enhancing the MPR list of a node depending on the load of links (i.e. the weights of links). We use the Algorithm described in Figure. 3 by flow chart to select the MPR set.

Notice that the ultimate goal of our proposed scheme is to find an MPR set smaller (or equal) to that selected in OLSR standard and ensure a higher QoS.

2) Second step: after selection of the MPR nodes according to our specifications, we calculate the path from the source to the destination pair in the network. Afterwards, we choose the path which offers the higher bandwidth. To this end, we modify the algorithm of Djikstra to take into account the parameter "weight" and do not necessarily focus on the shortest path. As a conclusion, the best path is expected to include links with large weights and it may be longer than that chosen in OLSR standard. This is because our main focus is the bandwidth offered by the path rather than the number of hops separating the source and destination nodes.

\section{Simulation SETTINGS AND RESUltS}

Having described the details of our proposed approach, we now focus on evaluating its performance through computer simulations using OPNET Modeler 14.0.

The main goal of this simulation is to study the efficiency of our algorithm and highlight its supremacy over the MPR selection heuristic used in the standard OLSR [1]. To this end, we have simulated the network topology shown in Figure. 8 and configured the routing, MAC and Traffic generation parameters as described in Table. I.

To highlight the effectiveness of our approach, two simulation scenarios are considered in the performance evaluation so as to illustrate the advantages of using the bandwidth estimation based scheme in OLSR. In the first scenario, we apply the MPR selection algorithm used in OLSR standard whereas in the second scenario our scheme is used. The obtained results in both scenarios are compared according to four different metrics which are; the average number of MPR nodes selected in the network, the induced overhead, the average throughput in the network and the network load.

\begin{tabular}{|l|l|}
\hline Parameters & Values \\
\hline \hline Area & $1000 \mathrm{~m} .1000 \mathrm{~m}$ \\
\hline Physical layer & Direct sequence \\
\hline Transmission range & $250 \mathrm{~m}$ \\
\hline Traffic & type CBR \\
\hline Routing protocol & OLSR \\
\hline Hello interval & 2 seconds \\
\hline TC interval & 5 seconds \\
\hline Topology & Random \\
\hline Data rate & 11 mbps \\
\hline CBR packets size & 500 bytes \\
\hline Simulation time & 600 seconds \\
\hline No. of simulation epochs & 5 \\
\hline Network simulator & OPNET 14.0 [10] \\
\hline
\end{tabular}

Table I: Simulation settings

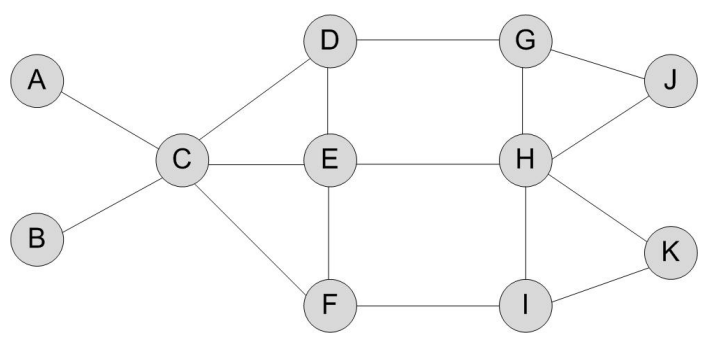

Figure 8: The topology used for evaluation of our proposed approach 


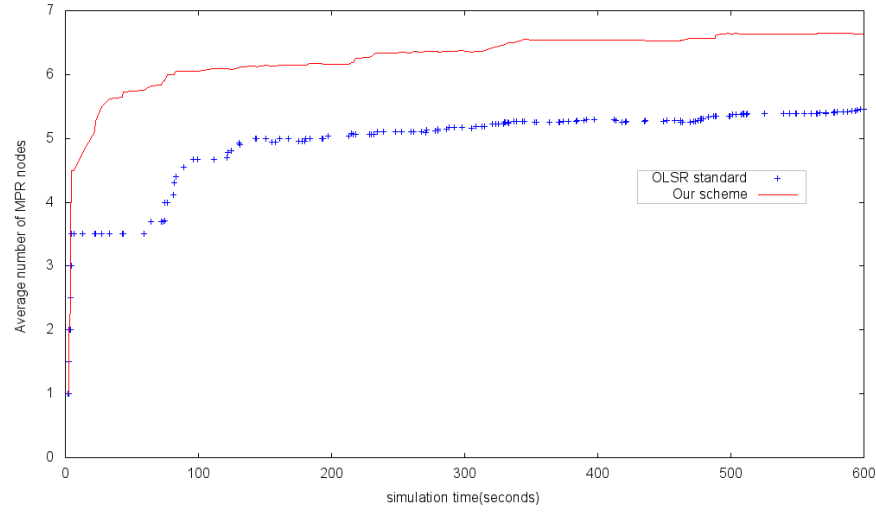

Figure 9: The average number of MPR nodes in the network: OLSR standard vs. our scheme

Figure. 9 shows the average number of MPR nodes selected in the network in both scenarios. We observe that the average number of the MPR nodes chosen in our approach (see the red curve) is greater than that chosen in OLSR standard which is represented by the blue curve. This increase is due to the selection of nodes that have a higher bandwidth rather than higher connectivity to improve the throughput in the network. Notice that the increase of the MPR-sets size in our scheme represents an advantage rather than drawback since it provides alternative routes in the network which might be useful in case of failure of some MPR nodes, especially in multi-path routing approaches. Moreover, these alternatives routes can be used to alleviate the burden of some MPR nodes due to their location in the network.

In Figure. 10, we compare the overhead induced by the transmission of control messages in OLSR Standard and the overhead added by the modified OLSR that uses our scheme. The higher routing overhead in our scheme is due to the increase of the number of MPR nodes in the network, as shown in Figure. 1. As the MPR nodes are the only nodes that generate, disseminate and forward the TC messages then the average number of TC messages sent in the network is proportional to the number of these MPR nodes. Obviously, the overhead added by our scheme is not negligible however the corresponding gain in bandwidth is more significant.

Figure. 11 shows clearly that the average throughput in the network has increased significantly in our scheme (see the red curve) compared to the throughput obtained in OLSR standard (see the blue curve). Notice that this rise of the throughput is stable as a consequence of the routing paths, chosen by our scheme, which provide higher bandwidth unlike those chosen in OLSR standard based on hops number which might, sometimes, hide the link with the best quality in terms of bandwidth.

The curves graphed on Figure. 12 reveal that the load in the network is much higher in our scheme (see the red curve) than the load measured in OLSR standard (see the blue curve). This increase is due to the fact that our scheme selects paths that carry more traffic than those selected by OLSR standard.

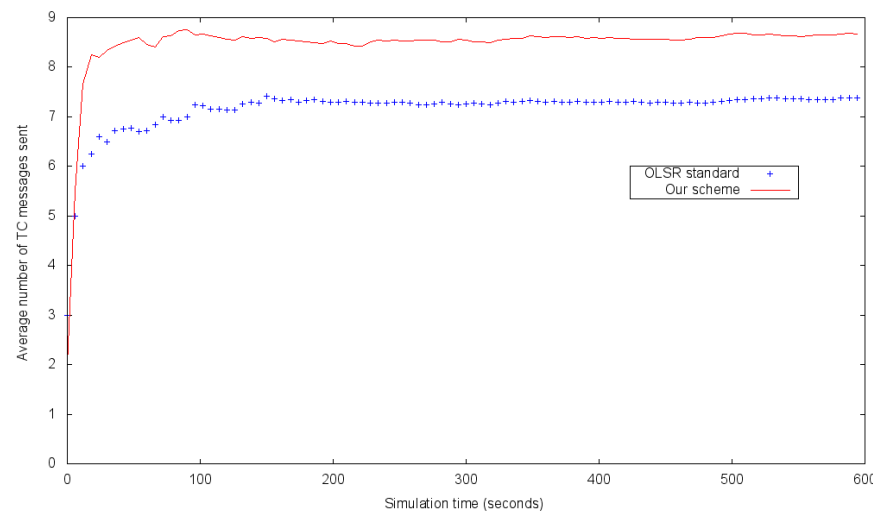

Figure 10: The overhead ( expressed as the average number of TC messages sent in the network) : OLSR standard vs. our scheme

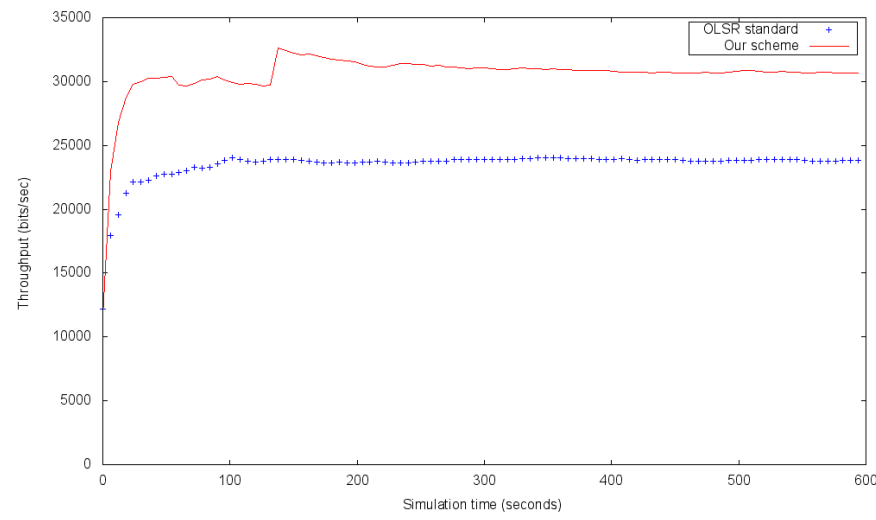

Figure 11: The throughput in the network: OLSR standard vs. our scheme

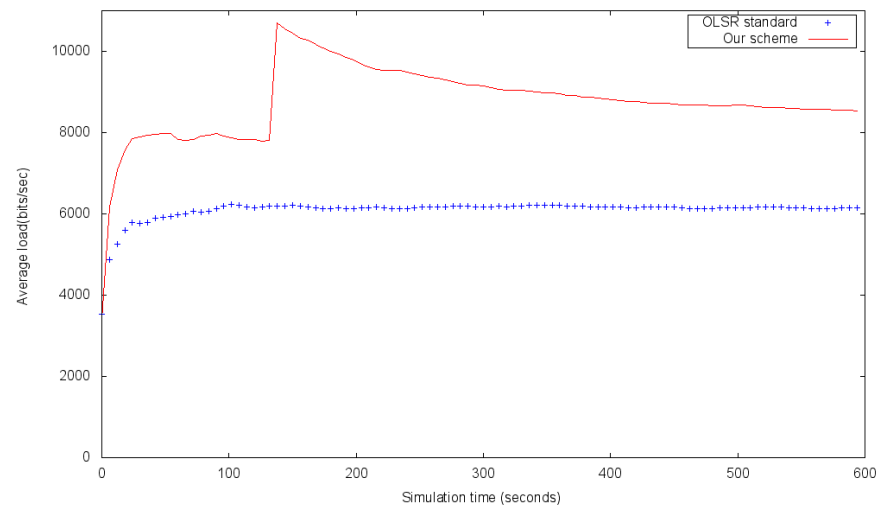

Figure 12: Load in the network: OLSR standard vs. our scheme 


\section{CONCLUSION}

In OLSR, the path selected to route traffic is based on MPRs with which the link with higher bandwidth can be hidden. In this paper we propose an approach to deal with this issue, our approach is a new scheme based on bandwidth estimation between adjacent nodes. To estimate the bandwidth, a conflict graph based approach has been used. After estimation the bandwidth, MPRs are selected taking inton account the link with higher bandwidth. Results from simulations have demonstrated that our scheme increased significantly the total throughput of WMNs. Moreover, it can also provides alternative routes with high bandwidth guarantees in case of failure of some MPR nodes.

\section{REFERENCES}

[1] T. Clausen and P. Jacquet. "RFC 3626: Optimized Link State Routing Protocol (OLSR)",Request for Comments, Category: Experimental, Project Hipercom, INRIA, IETF, Oct 2003.

[2] C. Perkins, E. Belding-Royer and S. Das, "Ad hoc On-Demand Distance Vector (AODV) Routing, IETF RFC 3561 (Experimental), July 2003.

[3] D. De Couto, D. Aguayo, J. Bicket, and R. Morris, "A high throughput path metric for mul ti-hop wireless routing, in Proc. MobiCom, 2003.

[4] I. F. Akyildiz, X. Wang, and W. Wang. ”Wireless Mesh Networks : A Survey", Comp. Networks, vol.47, no. 4, Mar. 2005, pp. 445-87.

[5] R. Draves J. Padhye B. Zill "Routing in Multi-Radio, Multi-Hop Wireless Mesh Networks", MobiCom04, Sept. 26-Oct. 1, 2004, Philadelphia, Pennsylvania, USA.

[6] Z. Fang and B. Bensaou. "Fair bandwidth sharing algorithms based on game theory frameworks for wireless ad hoc networks", in Proc. of IEEE INFOCOM 04,Hong Kong,Mar. 7-11, 2004.

[7] S. Djahel, F.Nait-abdesselam and D. Turgut. "Characterizing the Greedy Behavior in Wireless Ad Hoc Networks", Journal of Security and Communications Networks (SCN), Wiley InterScience, Vol. 4, No. 3, pp. 284-298, Mar. 2011.

[8] K. Jain, J. Padhye, V. Padmanabhan and L. Qiu. "Impact of Interference on Multi-hop Wireless Network Performance", in Proc. of the 9th Annual International Conference on Mobile Computing and Nteworking (MobiCom 03), Sun Diego, California, USA, Sep. 14-19, 2003.

[9] E. Tomita, A. Tanaka and H. Takahashi. "The worst-Case Time Complexity for Generating All Maximal Cliques", Theoritical Computer Science Journal of Elsevier, Vol. 363, No. 1, p 28-42, Oct. 2006.

[10] OPNET Technologies. OPNET Modeler. http://www.opnet.com/. 